DE

M E D I C I N A

T R O P I C A L

$\mathrm{DE}$

SÃO PAULO

JOURNAL OF THE SÃO PAULO INSTITUTE OF TROPICAL MEDICINE

${ }^{1}$ Wuhan University, Renmin Hospital, Department of Critical Care Medicine, Wuhan, Hubei Province, People's Republic of China

${ }^{2}$ Wuhan University, Renmin Hospital, Department of Respiratory and Critical Care Medicine, Wuhan, Hubei Province, People's Republic of China

Correspondence to: $\mathrm{Ke} \mathrm{Hu}$

Wuhan University, Renmin Hospital, Department of Respiratory and Critical Care Medicine, Wuhan 430060, Hubei Province, People's Republic of China

E-mail: huke-rmhospital@163.com, bigmousezuoxiaoshu@126.com

Received: 21 September 2020

Accepted: 2 February 2021

\section{Epidemiology and risk factors of candidemia due to Candida parapsilosis in an intensive care unit}

\author{
Xiao-shu Zuo ${ }^{(1)}$, , Yanan Liu ${ }^{1}, \mathrm{Ke} \mathrm{Hu}^{\left({ }^{\circledR} 2\right.}$
}

\section{ABSTRACT}

We analyzed the clinical features and risk factors of candidemia due to C. parapsilosis $(n=104)$ in the intensive care unit of a tertiary hospital over six years. This was a monocentric, retrospective study of candidemia, conducted from January 2013 to March 2019. Epidemiological characteristics, clinical features, invasive procedures, laboratory data and outcomes of 267 patients with candidemia were analyzed to determine risk factors of candidemia due to C. parapsilosis. Sixty-three cases of C. albicans and 204 cases of non-C. albicans Candida (NCAC) species were included, the latter was composed of 104 cases of $C$. parapsilosis and 100 cases of non-C. albicans species (46 cases of $C$. tropicalis, 22 cases of $C$. glabrata, 23 cases of $C$. guilliermondii, 5 cases of $C$. krusei and 4 cases of C. lusitaniae), suggesting that $C$. parapsilosis was the predominant $C$ andida species isolated from cases of candidemia. A binary multivariate logistic regression analysis showed that APACHE II scores, central venous catheterization and the use of broad-spectrum antibiotics were closely related to $C$. parapsilosis candidemia, with OR values of 1.159, 3.913 and 2.217, respectively. In conclusion, we found that $C$. parapsilosis was the main pathogen among the NCAC candidemia in the ICU patients. APACHE II scores, central venous catheterization and the use of broad-spectrum antibiotics were independent risk factors for the occurrence of $C$. parapsilosis candidemia, which may provide data to support the early introduction of anti-fungal therapy.

KEYWORDS: Candida parapsilosis. Candida spp. Candidemia. Risk factor.

\section{INTRODUCTION}

Candida albicans (C. albicans) is the most common known pathogen of invasive infections in adult non-neutropenic Intensive Care Unit (ICU) patients, causing roughly $70 \%$ of candidemia ${ }^{1,2}$, increasing the length of stay in either the ICU or the hospital, with an attributable mortality rate varying from $35 \%$ to $50 \%{ }^{3,4}$. However, in recent years, there has been a gradually increase in the incidence of candidemia caused by non-C. albicans species ${ }^{5-7}$, especially of Candida parapsilosis (C. parapsilosis $)^{8-10}$, which is reported as the second most commonly isolated Candida spp. from blood cultures (Figure 1), even outranking C. albicans and becoming the most important pathogens in candidemia cases ${ }^{11,12}$. More and more attention has been paid on its clinical features and pathogenesis by healthcare workers and experts.

Some studies suggest that the increasing incidence of $C$. parapsilosis candidemia is related to the characteristics of the treatment regimen (such as long-term hospitalization), microorganism-related properties (its specific affinity with the surface of intravascular devices and certain prosthetic materials or plastics to form 
Year 2010-2014 distribution of different Candida species from blood

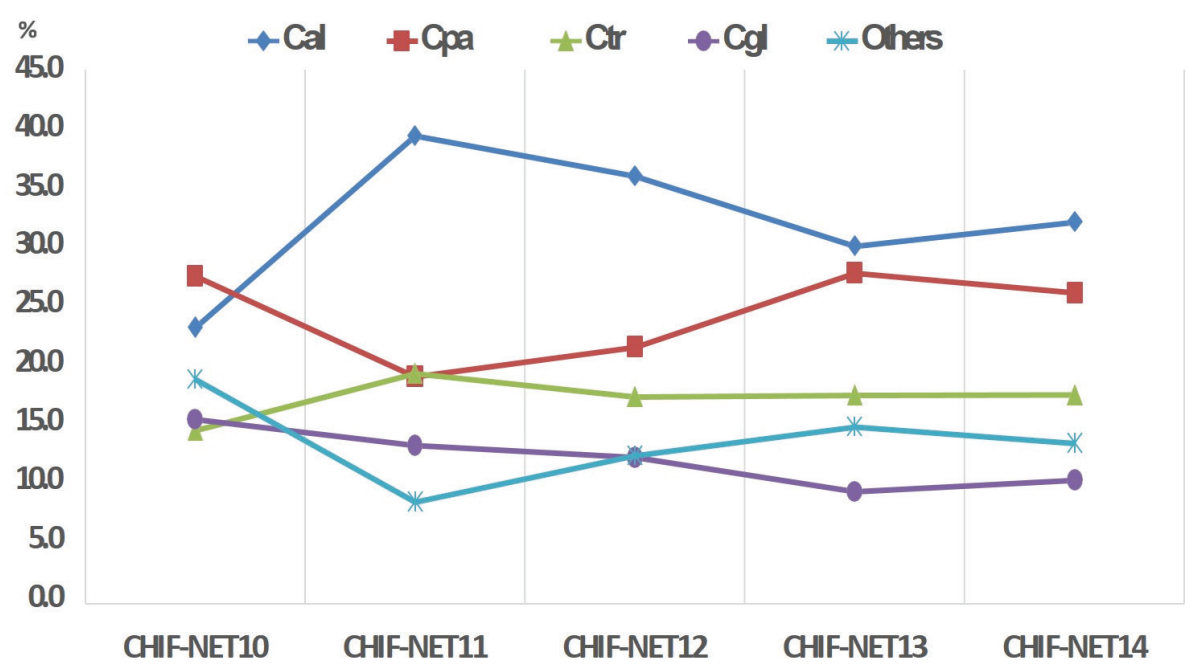

Figure 1 - Summary of 2014 data of CHIF-NET. The graphic indicated the incidence of different Candida species from blood in 5 years (2010-2014) published in China-Hospital-Invasive-Fungal-Surveillance-NET.

biofilms), and high glucose and fat environments favoring the gastrointestinal colonization and transmission ${ }^{5,13-16}$. Adhesion and biofilm formation are very important factors for $C$. parapsilosis indwelling on devices which appear to be the predominant route of infection ${ }^{15}$. The majority of patients with $C$. parapsilosis candidemia reported so far presented with underlying co-morbidities that required the use of intravenous indwelling catheters and total parenteral nutrition ${ }^{16}$. However, most of the studies were frequently small in size and covered a short period of time. Because of the increasing trend and the importance of $C$. parapsilosis to healthcare, our study intended to determine the risk factors of $C$. parapsilosis candidemia compared with those due to $C$. albicans and other non-C. albicans species in ICU wards of a large tertiary hospital in Hubei province, China.

\section{MATERIALS AND METHODS}

\section{Study design}

Clinical data were collected from patients with candidemia in ICU wards of a large tertiary hospital in Hubei, China, from January 2013 to March 2019. A total of 267 patients with candidemia were enrolled. The study protocol was reviewed and approved by the Committee for the Protection of Human Subjects at the Renmin Hospital of Wuhan University (RHWU).

\section{Definitions}

All cases met the diagnostic criteria of the Centers for Disease Control and Prevention (CDC ${ }^{17}$. They were reported by the Department of Clinical Microbiology Laboratory, and the species causing candidemia were identified using the IVD MALDI Biotyper mass spectrometry (Bruker, Germany). Inclusion criteria were: (1) A patient who developed signs or symptoms of blood stream infection (BSI) $>48 \mathrm{~h}$ after ICU admission. (2) Laboratory microbiological examination with at least one positive blood culture to Candida spp. and samples to perform blood cultures drawn from either a central venous catheter or a peripheral vein/artery. If the same patient had multiple episodes of candidemia during the study period, only the first episode of candidemia was included. (3) Presence of other signs or symptoms suggestive of systemic infections: fever $>38{ }^{\circ} \mathrm{C}$, chills, hypotension, multiple organ dysfunction, etc. Exclusion criteria: (1) Age $<18$ years; (2) Suspected candidemia occurred before ICU admission; (3) Transfer, death, or central venous catheter duration $<48 \mathrm{~h}$ after ICU admission.

\section{Data collection for risk factors}

In our study, 267 candidemia patients hospitalized in the ICU were included. All patients were retrospectively assessed for risk factors associated with candidemia. For all the identified patients, we performed a detailed medical record review and retrieved data from the electronic medical records and reports from the RHWU Clinical Microbiology Laboratory. Briefly, we need to collect demographic data, clinical data such as host factors, medical interventions and laboratory data. Host factors included age, gender, underlying diseases (comorbidities) such as lung disease, circulatory system disease, neoplastic 
disease, kidney disease, liver disease, etc., the APACHE II Score (Acute physiology and chronic health evaluation) within $24 \mathrm{~h}$ after ICU admission, ICU length of stay, and outcome. Retrieved medical interventions included total parenteral nutrition (TPN), mechanical ventilation (MV), prior use of medications (broad-spectrum antibiotics, hormones, immunosuppressants, etc.), abdominal surgeries, intravascular catheterization location (jugular, subclavian and femoral) and duration ( $>5$ days) considering the risks of infections and the time required by Candida spp. to adhere, colonize and form mature biofilms. Laboratory data included the patients' immune status (presence or absence of neutropenia), $\mathrm{G}$ test results, coinfections with bacteria and different organs disfunctions.

\section{Statistical analysis}

Categorical variables were compared by the chi square test or the Fisher exact test (SPSS Version 20.0, IBM, Armonk, NY, USA). Odds ratios (OR) and 95\% confidence intervals (CI) were calculated to evaluate the strength of selected associations. Continuous variables were compared using the Wilcoxon rank sum test. A two-tailed $p$-value of $<0.05$ was considered significant. A multivariate logistic regression analysis was performed to identify associations between variables and risk factors to develop candidemia due to $C$. parapsilosis compared to other Candida species. Besides the multi-collinearity among some variables in the multivariate analysis, variables with a univariate $p$-value $<0.1$ were considered for the inclusion in the multivariate model.

\section{RESULTS}

During the 6-year study period 2013-2019, 267 candidemia patients were enrolled at RHWU (Figure 2). All the included patients were divided into groups: $C$. albicans, C. parapsilosis and other non-C. albicans species group.

\section{Species distribution}

Among the detected 267 patients with candidemia, $63(23.6 \%)$ were caused by C. albicans, $104(39.0 \%)$ by C. parapsilosis and 100 (37.4\%) by other non-C. albicans species, include $46(17.2 \%)$ cases of $C$. tropicalis, $22(8.2 \%)$ cases of C. glabrata, $23(8.6 \%)$ cases of C. guilliermondii, 5 (1.9\%) cases of C. krusei and 4 (1.5\%) cases of $C$. lusitaniae (Table 1). The total number of isolates of non-C. albicans species was 204 (76.4\%). The most common isolated species were $C$. parapsilosis, followed by $C$. albicans, $C$. tropicalis, $C$. guilliermondii, C. glabrata and C. krusei.

Table 1 - Distribution of different Candida species.

\begin{tabular}{cc}
\hline Species & Number (\%) \\
\hline C. albicans & $63(23.6)$ \\
C. parapsilosis & $104(39.0)$ \\
Other non-Candida albicans species & $100(37.4)$ \\
C. tropicalis & $46(17.2)$ \\
C. glabrata & $22(8.2)$ \\
C. guilliermondii & $23(8.6)$ \\
C. krusei & $5(1.9)$ \\
C. lusitaniae & $4(1.5)$ \\
\hline
\end{tabular}

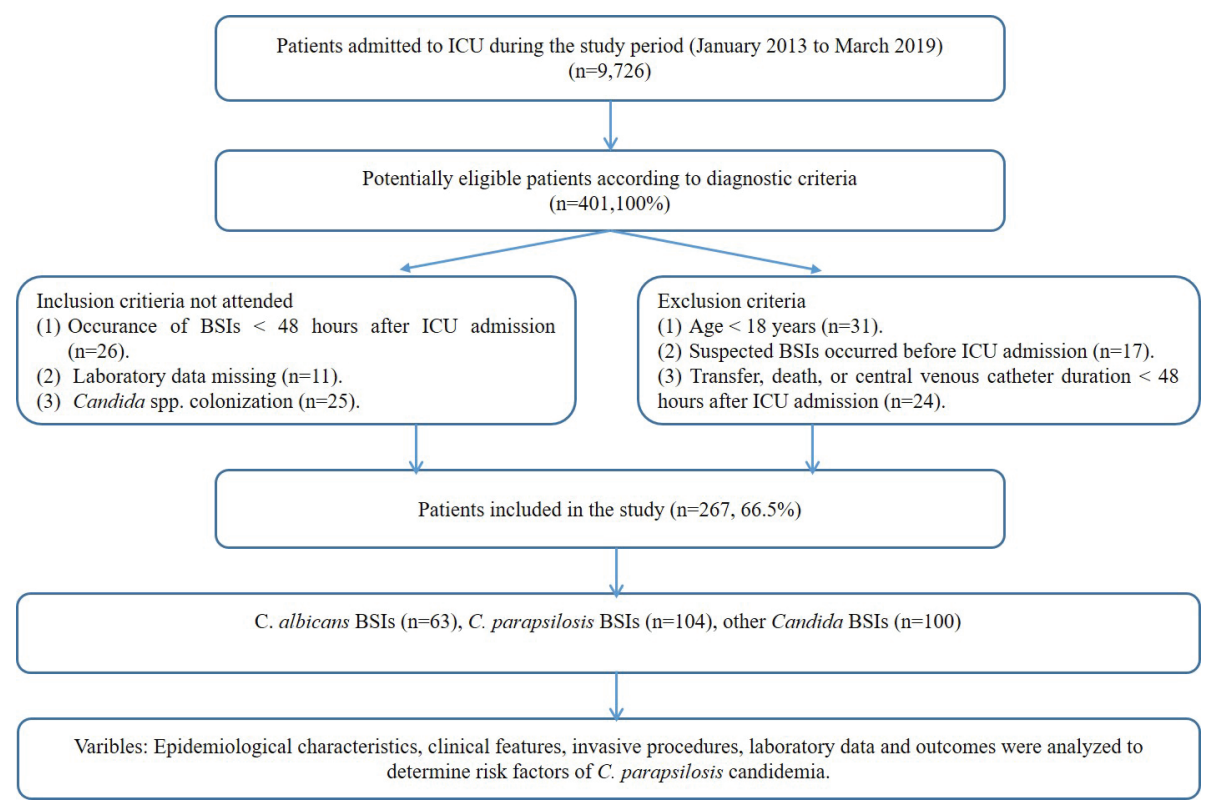

Figure 2 - Flow diagram. The graphic described the diagnostic criteria, inclusion criteria and exclusion criteria, how many patients were included and explained why. 
Demographic characteristics of patients with different species of candidemia are shown in Table 2. One hundred and twenty one $(45.3 \%)$ patients were male and the mean age of patients was $58.12 \pm 16.51$ years. In the $C$. albicans group, 33 patients were male and 30 patients were female, the mean age was $61.41 \pm 15.98$ years, while in the non- $C$. albicans species group, 88 (33.0\%) patients were male and 116 (43.4\%) patients were female, and the mean age was 57.11 \pm 16.58 years. Regarding gender and age, no significant differences were found between patients with candidemia due to $C$. parapsilosis and other Candida species.

To investigate the correlation between severity of illness and episodes of candidemia caused by $C$. parapsilosis, we found that the mean of APACHE II Scores in all groups was above 20. In the $C$. albicans group, the mean of APACHE II Score was $21.71 \pm 3.21 ; 25.85 \pm 6.08$ in the $C$. parapsilosis group and $22.33 \pm 4.90$ in the group of other non-C. albicans species. We found that the APACHE II Score of patients with $C$. parapsilosis candidemia episodes was higher than in both groups of $C$. albicans and other non-C. albicans species $(p=0.000)$. These results are shown in Table 2 .

Relationship between the presence of underlying diseases (comorbidities) and episodes of candidemia caused by different Candida species

We investigated the presence of underlying diseases (comorbidities) and the main diagnosis of the enrolled ICU patients. There were 16 cases of type 2 diabetes, 14 cases of renal insufficiency, 20 patients with hypertension, 13 cases of heart disease, 10 cases of chronic obstructive pulmonary disease, 13 cases of cerebral ischemia, 3 cases of cirrhosis, 3 cases of congenital heart disease, 8 cases of neoplastic disease, 2 cases of autoimmune disease and 35 cases of comorbidities involving more than 2 systems. There were no significant differences in the frequency of comorbidities and the major causes of ICU admission among groups of candidemia by $C$. albicans, $C$. parapsilosis and other non-C. albicans species. These results are shown in Table 2.

Relationship between the use of medical interventions and the presence of episodes of candidemia caused by different Candida species

Of the 267 patients, 124 (46.4\%) cases were placed with a central venous catheter (double-lumen), of which 21 cases were in C. albicans group, 75 cases were in C. parapsilosis group, and 28 cases were in other non-C. albicans species group. Regarding the catheter location, there were 48 cases of subclavian vein catheterization (six cases in C. albicans group, 32 cases in C. parapsilosis group, and 10 cases in other groups), 35 cases of internal jugular vein catheterization (ten cases in $C$. albicans group, 16 cases in C. parapsilosis group, and nine cases in other groups), 57 cases of femoral vein catheterization (nine cases in C. albicans group, 34 cases in C. parapsilosis group,

Table 2 - Comparison of epidemiological characteristics of patients hospitalized in an intensive care unit presenting with infections caused by different species of Candida.

\begin{tabular}{|c|c|c|c|c|}
\hline Variables ${ }^{*}$ & $\begin{array}{l}\text { C. albicans } \\
(n=63)\end{array}$ & $\begin{array}{c}\text { C. parapsilosis } \\
(\mathrm{n}=104)\end{array}$ & $\begin{array}{l}\text { Others" } \\
(n=100)\end{array}$ & $P$ \\
\hline Age (years) (Mean \pm SD) & $61.41 \pm 15.98$ & $57.02 \pm 15.51$ & $57.20 \pm 17.69$ & $>0.05$ \\
\hline Gender (Male) & $33(52.4)$ & 46 (44.2) & $42(42.0)$ & $>0.05$ \\
\hline Comorbidities & $25(39.7)$ & $33(31.7)$ & $44(44.0)$ & \\
\hline Type 2 diabetes & $4(6.3)$ & $5(4.8)$ & $7(7.0)$ & \\
\hline Renal insufficiency & $4(6.3)$ & $4(3.8)$ & $6(6.0)$ & \\
\hline Hypertension & $5(7.9)$ & $6(5.8)$ & $9(9.0)$ & \\
\hline Coronary heart disease & $4(6.3)$ & $4(3.8)$ & $5(5.0)$ & \\
\hline COPD & $2(3.2)$ & $4(3.8)$ & $4(4.0)$ & \\
\hline Cerebral ischemia & $2(3.2)$ & $5(4.8)$ & $6(6.0)$ & $>0.05$ \\
\hline Cirrhosis & - & - & $3(3.0)$ & \\
\hline Congenital heart disease & $2(3.2)$ & $1(0.96)$ & - & \\
\hline Neoplastic disease & $2(3.2)$ & $3(2.9)$ & $3(3.0)$ & \\
\hline Autoimmune disease & - & $1(0.96)$ & $1(1.0)$ & \\
\hline$>2$ systems diseases & $9(14.3)$ & $15(14.4)$ & $11(11.0)$ & \\
\hline APACHE II Score & $21.71 \pm 3.21$ & $25.85 \pm 6.08^{a}$ & $22.33 \pm 4.90$ & 0.000 \\
\hline ICU length of stay (days) & $14.38 \pm 8.05$ & $14.13 \pm 7.50$ & $12.98 \pm 7.73$ & $>0.05$ \\
\hline Mortality & $5(7.9)$ & $12(11.5)^{a}$ & $8(8.0)$ & 0.046 \\
\hline
\end{tabular}

"Values for all variables except age, APACHE II Scores and ICU-stay are given in percentages of patients; "All Candida species other than C. albicans or C. parapsilosis included 46 C. tropicalis, 22 C. glabrata, 23 C. guilliermondii, 5 C. krusei and 4 C. Iusitaniae; $\mathrm{SD}=$ Standard deviation; APACHE = Acute Physiology and Chronic Health Evaluation; ICU = Intensive Care Unit; COPD = Chronic obstructive pulmonary disease; ${ }^{a} P<0.05$, significant difference between between $C$. parapsilosis and Others (C. parapsilosis vs Others). 
14 cases in other groups), and eight cases of basilic vein catheterization (one case in C. albicans group, five cases in C. parapsilosis group, two cases in other groups). Among them, 24 patients had catheters in both, the jugular vein or the subclavian vein or the basilic vein and the femoral vein (four cases in C. albicans group, 13 cases in C. parapsilosis group, and seven cases in non-C. albicans species group). We found that the presence of central venous catheters, the CVC duration $>5$ days and the catheterization locations in the subclavian and the femoral vein, were significant different between $C$. parapsilosis, C. albicans and the other non- $C$. albicans species group $(p=0.000 ; 0.024 ; 0.000$; 0.002). These results are shown in Table 3.

It has been reported that total parenteral nutrition (TPN) is an important factor of biofilm formation. We examined the total parenteral nutrition $>3$ days in the different Candida species groups and found that differences were significant between C. parapsilosis, C. albicans and other non-C. albicans species $(p=0.000)$. These results are shown in Table 3.

In respect to the prior risk factors (reported before the ICU hospitalization), we checked the use of broad-spectrum antibiotics in the different Candida species groups and found that there was a significant difference between C. albicans and other non-C. albicans species $(p=0.000)$, also between $C$. albicans and $C$. parapsilosis $(p=0.000)$. Considering the mechanical ventilation, there was a significant difference between C. parapsilosis, C. albicans and other non-C. albicans species ( $p=0.000 ; 0.046)$ groups. These results are shown in Table 3.

\section{Comparison of laboratory data of different species causing candidemia}

We investigated some important clinical parameters including neutropenia, positivity of the $\mathrm{G}$ test $(1,3-\beta$-DGlucan [BDG]) test. which is a fungal cell wall constituent used in the diagnosis of invasive fungal infections), the presence of multiple organ dysfunction syndrome (MODS) and coinfections. Through these comparisons, we found that there were no significant differences among groups of $C$. albicans, $C$. parapsilosis and other non-C. albicans species. These results are shown in Table 4.

\section{Risk factors associated with C. parapsilosis candidemia}

Relevant risk factors included in the analysis of C. parapsilosis bloodstream infections were gender, age, underlying diseases (comorbidities), the APACHE II score, the ICU length of stay (days), mortality, use of broad-spectrum antibiotics, use of steroids and immunosuppressants, CVC placement and location, catheterization duration $>5$ days, use of total parenteral nutrition (TPN) $>3$ days, special medical interventions such as continuous renal replacement therapy (CRRT), mechanical ventilation, abdominal surgeries, clinical data

Table 3 - Comparison of the relationship between different species of Candida infections and medical interventions.

\begin{tabular}{|c|c|c|c|c|c|}
\hline Variables ${ }^{*}$ & $\begin{array}{l}\text { C. albicans } \\
(n=63)\end{array}$ & $\begin{array}{l}\text { C. parapsilosis } \\
(\mathrm{n}=104)\end{array}$ & Others" $(n=100)$ & $95 \% \mathrm{Cl}$ & $P$ \\
\hline Broad-spectrum antibiotics & $30(47.6)^{a}$ & $76(73.1)$ & $73(73.0)$ & $0.338-0.554$ & 0.000 \\
\hline Steroids & $2(3.2)$ & $4(3.8)$ & $5(5.0)$ & - & $>0.05$ \\
\hline Immunosuppressants & $0(0.0)$ & $0(0.0)$ & $1(1.0)$ & - & - \\
\hline Central venous catheter & $21(33.3)$ & $75(72.1)^{b}$ & $28(28.0)$ & $0.277-0.530$ & 0.000 \\
\hline Internal jugular & $10(15.9)$ & $16(15.4)$ & $9(9.0)$ & - & $>0.05$ \\
\hline Subclavian vein & $6(9.5)$ & $32(30.8)^{b}$ & $10(10.0)$ & 0.099-0.316 & 0.000 \\
\hline Femoral vein & 9 (14.3) & $34(32.7)^{b}$ & $14(14.0)$ & $0.072-0.302$ & 0.002 \\
\hline Basilic vein & $1(1.6)$ & $5(4.8)$ & $2(2.0)$ & - & $>0.05$ \\
\hline CVC duration $>5$ days & $14(22.2)$ & $45(43.3)^{b}$ & $23(23.0)$ & $0.019-0.271$ & 0.024 \\
\hline Total parenteral nutrition $>3$ days & $21(33.3)$ & $72(69.2)^{b}$ & $42(42.0)$ & $0.140-0.405$ & 0.000 \\
\hline Mechanical ventilation & $30(47.6)^{a}$ & $69(66.3)^{\mathrm{b}}$ & $32(32.0)$ & $\begin{array}{l}0.003-0309 \\
0.213-0.474\end{array}$ & $\begin{array}{l}0.046 \\
0.000\end{array}$ \\
\hline Continuous renal replacement therapy & $11(17.5)$ & $21(20.2)$ & $13(13.0)$ & - & $>0.05$ \\
\hline Abdominal surgeries & $16(25.4)$ & $27(26.0)$ & $26(26.0)$ & - & $>0.05$ \\
\hline
\end{tabular}

"Values for all variables are given in percentages of patients; "All Candida species other than C. albicans or C. parapsilosis included 46 C. tropicalis, 22 C. glabrata, 23 C. guilliermondii, 5 C. krusei and 4 C. lusitaniae; CVC = Central venous catheter; ${ }^{a} P<0.05$, significant difference between $C$. albicans and other Candida species (C. albicans vs Others); ${ }^{b} P<0.05$, significant difference between C. parapsilosis and other Candida species (C. parapsilosis vs Others). 
Table 4 - Comparison of laboratory data of different species of Candida causing infections.

\begin{tabular}{lcccc}
\hline Variables* & $\begin{array}{c}\text { C. albicans } \\
(\mathrm{n}=63)\end{array}$ & $\begin{array}{c}\text { C. parapsilosis } \\
(\mathrm{n}=104)\end{array}$ & $\begin{array}{c}\text { Others\# } \\
(\mathrm{n}=100)\end{array}$ & $P$ \\
\hline Neutropenia & $0(0.0)$ & $3(2.8)$ & $0(0.0)$ & $>0.05$ \\
Positive G test & $25(39.7)$ & $39(37.5)$ & $38(38.0)$ & $>0.05$ \\
MODS & $20(31.7)$ & $35(33.7)$ & $41(41.0)$ & $>0.05$ \\
Coinfection & $28(44.4)$ & $38(36.5)$ & $39(39.0)$ & $>0.05$ \\
\hline
\end{tabular}

"Values for all variables are given in percentages of patients; $A$ All Candida species other than C. albicans or C. parapsilosis included 46 C. tropicalis, 22 C. glabrata, 23 C. guilliermondii, 5 C. krusei and 4 C. lusitaniae; MODS = Multiple organ dysfunction syndrome.

such as neutropenia, positivity of the $\mathrm{G}$ test, the multiple organ dysfunction syndrome (MODS), coinfections with other bacteria (mainly Gram-positive / negative bacteria).

An univariate analysis was performed on the variables, searching for possible risk factors of $C$. parapsilosis bloodstream infections. The results showed that the APACHE II Score, therapy with broad-spectrum antibiotics, the presence of a central venous catheter, catheterization locations and duration, total parenteral nutrition $>3$ days and mechanical ventilation were all risk factors for C. parapsilosis bloodstream infection $(p=0.000 ; 0.001$; $0.000 ; 0.003 ; 0.013 ; 0.009 ; 0.000 ; 0.029)$. Regarding to the ICU length of stay, CRRT treatment, abdominal surgeries, neutropenia, a positive G test, MODS and coinfections were not significantly related to the occurrence of $C$. parapsilosis bloodstream infections ( $p>0.05)$. Results are shown in Table 5. To further analyze variables in the univariate analysis (Table 5), a multivariate regression analysis was performed. Considering multi-collinearity in the multivariate analysis, it was found that mechanical ventilation was a confounding factor $(p>0.05)$, while the APACHE II score, CVC catheterization, and the use of broad-spectrum antibiotics were closely related to $C$. parapsilosis bloodstream infections $(p=0.001$;

Table 5 - Risk factors for $C$. parapsilosis bloodstream infections after single parameter analysis.

\begin{tabular}{|c|c|c|c|c|}
\hline & $\mathrm{B}$ & Wald & OR $(95 \% \mathrm{Cl})$ & $P$ \\
\hline Age (year) (Mean \pm SD) & -0.015 & 2.184 & $0.985(0.965-1.005)$ & 0.139 \\
\hline Gender (Male) & -0.275 & 0.733 & $0.760(0.405-1.425)$ & 0.392 \\
\hline Underlying comorbidities & -0.277 & 0.687 & $0.758(0.394-1.459)$ & 0.407 \\
\hline APACHE II Scores & 0.164 & 19.157 & $1.179(1.095-1.268)$ & 0.000 \\
\hline ICU length of stay (days) & -0.008 & 0.156 & $0.992(0.952-1.033)$ & 0.693 \\
\hline Mortality & 0.386 & 0.478 & $1.471(0.493-4.393)$ & 0.498 \\
\hline Central venous catheter & 1.585 & 21.178 & $4.881(2.485-9.588)$ & 0.000 \\
\hline Internal jugular catheter & -0.067 & 0.024 & $0.935(0.395-2.211)$ & 0.878 \\
\hline Subclavian vein catheterization & 1.409 & 8.650 & $4.091(1.600-10.462)$ & 0.003 \\
\hline Femoral vein catheterization & 1.037 & 6.196 & $2.820(1.247-6.739)$ & 0.013 \\
\hline Basilic vein catheterization & 1.115 & 1.014 & $3.050(0.348-26.727)$ & 0.314 \\
\hline Broad-spectrum antibiotics & 1.141 & 11.468 & $3.129(1.617-6.056)$ & 0.001 \\
\hline Steroids & 0.172 & 0.038 & $1.188(0.211-6.683)$ & 0.845 \\
\hline CVC duration $>5$ days & 0.944 & 6.801 & $2.571(1.264-5.229)$ & 0.009 \\
\hline Total parenteral nutrition $>3$ days & 1.449 & 18.074 & $4.260(2.184-8.309)$ & 0.000 \\
\hline Mechanical ventilation & 0.715 & 4.768 & $2.044(1.077-3.880)$ & 0.029 \\
\hline Continuous renal replacement therapy & 0.148 & 0.128 & $1.159(0.517-2.601)$ & 0.720 \\
\hline Abdominal surgeries & -0.005 & 0.000 & $0.995(0.486-2.040)$ & 0.989 \\
\hline Positive G test & -0.134 & 0.167 & $0.875(0.459-1.664)$ & 0.683 \\
\hline MODS & 0.049 & 0.020 & $1.050(0.538-2.051)$ & 0.886 \\
\hline Coinfection & -0.267 & 0.668 & $0.766(0.404-1.452)$ & 0.414 \\
\hline
\end{tabular}

$\mathrm{B}$ = Coefficient estimates; Wald: Chi-square value; OR: Odds ratio; $\mathrm{Cl}=$ Confidence interval; $\mathrm{APACHE}=\mathrm{Acute}$ Physiology and Chronic Health Evaluation; CVC = Central venous catheter. 
Table 6 - Risk factors in the multivariate analysis of C. parapsilosis bloodstream infections.

\begin{tabular}{lcccc}
\hline & B & Wald & OR (95\% Cl) & $P$ \\
\hline APACHE II Scores & 0.148 & 11.969 & $1.159(1.066-1.261)$ & $\mathbf{0 . 0 0 1}$ \\
Central venous catheter & 1.364 & 11.978 & $3.913(1.807-8.474)$ & $\mathbf{0 . 0 0 1}$ \\
Broad-spectrum antibiotics & 0.796 & 4.233 & $2.217(1.038-4.734)$ & $\mathbf{0 . 0 4 0}$ \\
\hline
\end{tabular}

$\mathrm{B}=$ Coefficient estimates; Wald = Chi-square value; $\mathrm{OR}=$ Odds ratio; $\mathrm{Cl}=$ Confidence interval; $\mathrm{APACHE}=\mathrm{Acute}$ Physiology and Chronic Health Evaluation.

$0.001 ; 0.040$ ), with OR values of $1.159,3.913$ and 2.217 , respectively. Results are shown in Table 6.

\section{Treatment and prognosis}

The main treatment measures included removal of the central venous catheters and antifungal therapy. Echinocandins (micafungin, caspofungin) were used as previously recommended ${ }^{3}$. In two cases, the central catheters could not be removed or replaced. There were 25 deaths (five in C. albicans group, 12 in C. parapsilosis group, and eight in the other non-C. albicans groups). There was a statistical difference in mortality between the C. parapsilosis group and the other non-C. albicans species group $(p=0.046)$, while there were no statistical differences in ICU length of stay between the groups. These results are shown in Table 2.

\section{DISCUSSION}

Candida bloodstream infections are still serious nosocomial infections worldwide and are the third or fourth causes of healthcare-related bloodstream infections ${ }^{8}$. C. albicans has been regarded as the main pathogenic fungus, however, in recent years, non-C. albicans species have gradually become the main pathogen of hospitalacquired candidemia ${ }^{11,12}$. Studies have shown that the most frequently implicated risk factors of invasive candidemia include the use of broad-spectrum antibiotics, immunossupressant agents (glucocorticosteroids, chemotherapeutic agents, and immunomodulators), neutropenia, use of central venous catheters, total parenteral nutrition, renal replacement therapy and use of implantable prosthetic devices $^{18,19}$. A number of studies have shown that the rate of C. parapsilosis isolation from blood samples or venous catheter implants is second to or higher than the rate of C. albicans isolation from the same source ${ }^{20,21}$. In our study, a total of 267 patients with candidemia were included. Among them, C. parapsilosis accounted for $38.95 \%$ (104 cases) of candidemia cases, while the proportion of C. albicans was $23.60 \%$ (63 cases) and of other non-C. albicans species was $37.45 \%$ (100 cases) (Table 1$)$.
To our surprise, the proportion of candidemia attributed to C. parapsilosis was even higher than the one of $C$. albicans during our study period, indicating that non-C. albicans species are becoming the most common cause of Candida bloodstream infections, which is consistent with data of previous studies ${ }^{11,12,21,22}$.

Several studies ${ }^{6,8}$ have shown that the occurrence of candidemia is associated with increasing length of hospital stay and medical costs. Almirante et al. ${ }^{22}$ found that the early and overall mortality of patients with $C$. parapsilosis candidemia was lower than those of C. albicans (6\% vs $25 \%$, 7 days; $23 \%$ vs $43 \%$, overall; $p<0.01$ ). In our study, we compared candidemia caused by different species andthe results indicated that there was no significant differences in terms of mortality and ICU length of stay compared to candidemia caused by $C$. parapsilosis and C. albicans.

Regarding risk factors, an observational study in Europe concluded that catheter implantation, use of broadspectrum antibiotic, total parenteral nutrition, abdominal surgeries, glucocorticoid therapy, tumors, organ transplants, neutropenia and previous Candida colonization are highrisk factors for $C$. parapsilosis candidemia ${ }^{22}$. Our study showed that APACHE II score, CVC catheterization and use of broad-spectrum antibiotics were independent risk factors for $C$. parapsilosis candidemia (Table 6).

APACHE II is a severity-of-disease classification system proposed by Knaus et al..$^{23}$ that has been widely used in emergency and critical care medicine. It is determined within $24 \mathrm{~h}$ of admission to an ICU, with higher scores corresponding to more severe disease and a higher risk of death. In our study, the average APACHE II score of C. parapsilosis candidemia patients was above 25 , which was significantly higher than the one of candidemia patients caused by other Candida species (Table 2). We can also imply that $C$. parapsilosis candidemia patients were in more severe conditions when admitted to emergency department.

It is reported that approximately $80 \%$ of patients with non-neutropenic candidemia originated from the presence of intravascular catheters, such as a central venous catheter, a hemodialysis catheter, a PICC or an implantation port. Clinically useful biological materials can easily favor Candida colonization and biofilm 
formation, and cause related bloodstream infections ${ }^{24-27}$. In our study, patients with $C$. parapsilosis candidemia showed a higher rate of CVC compared with those of other Candida species (Table 3). An analysis of 393 cases of Candida spp. bloodstream infections in Switzerland, from 2005 to 2006, showed that the positive rate of biofilms in cases caused by non-C. albicans species $(88.7 \%)$ was significantly higher than that of C. albicans (40.3\%), while in the $C$. parapsilosis group the rate of biofilms reached $66.7 \%$. This study indicated that the ability of Candida to adhere to the surface of the conduct to form a biofilm was highly strain-dependent. C. parapsilosis isolated from different locations have different biofilmforming capabilities, which was the strongest when the isolates were from bloodstream cultures ${ }^{28}$. Studies by Branchini et al. ${ }^{14}$ found that $C$. parapsilosis can proliferate in high-concentration glucose conditions and form a biofilm on the prosthetic material. The mucus production by $C$. parapsilosis helps it to fix on plastic catheters, and the biofilm formation is related to the colonization ability of $C$. parapsilosis placed on the central venous catheter, and has been considered as a virulence factor for C. parapsilosis candidemia. A Costa Rican clinical study found a strong association between $C$. parapsilosis candidemia and central venous catheterization and total parenteral nutrition ${ }^{20}$. In addition, the colonization and C. parapsilosis infection depend on its ability to adhere to host cell tissues, especially mucosal epithelia. As adhesion can promote the destruction of adjacent host cells by Candida, this adhesion in C. parapsilosis may be related to the presence of hydrophobic molecules on the surface of Candida spp. ${ }^{29}$.

Our results indicated that the administration of broadspectrum antibiotics was another independent risk factors for C. parapsilosis candidemia (Table 6). We know that empiric broad-spectrum therapy with one or more intravenous antimicrobials to cover all likely pathogens should be started immediately in patients presenting with sepsis or septic shock ${ }^{30}$. In the updated Surviving Sepsis Campaign (SSC) bundle of care ${ }^{31}$, which have combined the 3-h and 6-h bundles into a single "hour-1 bundle" recommendations such as obtaining blood cultures prior to administration of antibiotics and administration of broad-spectrum antibiotics in $1 \mathrm{~h}$ should be accomplished within "hour-1 bundle". Once the pathogen identification and its sensitivities are established, empiric antimicrobial therapy should be narrowed or discontinued if infection is subsequently proven not to exist. The link between early administration of broad-spectrum antibiotics for suspected infection and antibiotic stewardship remains an essential aspect of high-quality sepsis management.
In short, this study analyzed the demographic characteristics, underlying comorbidities, clinical interventions and laboratory data of patients with candidemia indicating that among the pathogens that cause candidemia in ICU, C. parapsilosis among the non-C. albicans species is becoming dominant. Moreover, the results showed that APACHE II score, CVC catheterization and use of broadspectrum antibiotics were independent risk factors for C. parapsilosis candidemia. Because this is a monocentric study, further multicenter studies placed in different geographical regions should perform studies on candidemia in ICU patients to better define the epidemiology and pathogenesis of candidemia, helping intensive care specialists to assess the distribution and trends in their patients with clinically suspected candidemia.

\section{CONCLUSION}

In the ICU patients with candidemia, C. parapsilosis is becoming the main pathogen among NCAC candidemia. APACHE II scores, central venous catheterization and use of broad-spectrum antibiotics were independent risk factors for the occurrence of C. parapsilosis candidemia, which may provide data to support the early anti-fungal therapy. There are several limitations in the present study. First of all, this is a retrospective clinical study which is frequently used for severe nosocomial infections with low incidence rates that may need very large sample sizes for more accurate outcome analysis. Secondly, the sample in this study was selected over 6 years, so that the testing procedures may have varied over time. However, in our study, we have not detected any variation over time. Finally, this is a monocentric study and the findings are focused on $C$. parapsilosis candidemia, so that the results can be extrapolated but with caution. Further multicenter studies can better define the epidemiology and pathogenesis of C. parapsilosis candidemia.

\section{ACKNOWLEDGMENTS}

This work was supported by the National Science Foundation of China (Project $\mathrm{N}^{\circ}: 81801959$, Project name: The Role and mechanism of Tim-3/Galectin-9 cosuppression signal regulate $\mathrm{CD} 8+\mathrm{T}$ cells in liver immune tolerance induced by sepsis).

\section{ETHICAL APPROVAL}

The protocol was approved by the medical ethics committee of the Renmin Hospital, Wuhan University. 


\section{CONFLICT OF INTERESTS}

The authors declare that they have no conflict of interests.

\section{AUTHORS' CONTRIBUTIONS}

$\mathrm{KH}$ conceived the project and designed the study, XSZ and YNL analyzed and interpreted the data. XSZ drafted the first draft of the manuscript. All authors revised and approved the final version of the manuscript.

\section{REFERENCES}

1. Pfaller MA. Antifungal drug resistance: mechanisms, epidemiology, and consequences for treatment. Am J Med. 2012;125 Suppl 1:S3-13.

2. Silva-Dias A, Miranda IM, Branco J, Monteiro-Soares M, PinaVaz C, Rodrigues AG. Adhesion, biofilm formation, cell surface hydrophobicity, and antifungal planktonic susceptibility: relationship among Candida spp. Front Microbiol. 2015;6:205.

3. Pappas PG, Kauffman CA, Andes DR, Clancy CJ, Marr KA, Ostrosky-Zeichner L, et al. Clinical practice guideline for the management of candidiasis: 2016 update by the Infectious Diseases Society of America. Clin Infect Dis. 2016;62:e1-50.

4. Moran C, Grussemeyer CA, Spalding JR, Benjamin DK, Jr, Reed SD. Comparison of costs, length of stay, and mortality associated with Candida glabrata and Candida albicans bloodstream infections. Am J Infect Control. 2010;38:78-80.

5. van Asbeck EC, Clemons KV, Stevens DA. Candida parapsilosis: a review of its epidemiology, pathogenesis, clinical aspects, typing and antimicrobial susceptibility. Crit Rev Microbiol. 2009;35:283-309.

6. Pfaller MA, Diekema DJ, Gibbs DL, Newell VA, Ellis D, Tullio V, et al. Results from the ARTEMIS DISK Global Antifungal Surveillance Study, 1997 to 2007: a 10.5-year analysis of susceptibilities of Candida Species to fluconazole and voriconazole as determined by CLSI standardized disk diffusion. J Clin Microbiol. 2010;48:1366-77.

7. Cleveland AA, Farley MM, Harrison LH, Stein B, Hollick R, Lockhart SR, et al. Changes in incidence and antifungal drug resistance in candidemia: results from population-based laboratory surveillance in Atlanta and Baltimore, 2008-2011. Clin Infect Dis. 2012;55:1352-61.

8. Pfaller MA, Diekema DJ. Epidemiology of invasive candidiasis: a persistent public health problem. Clin Microbiol Rev. 2007;20:133-63.

9. Horn DL, Neofytos D, Anaissie EJ, Fishman JA, Steinbach WJ, Olyaei AJ, et al. Epidemiology and outcomes of candidemia in 2019 patients: data from the prospective antifungal therapy alliance registry. Clin Infect Dis. 2009;48:1695-703.
10. Conde-Rosa A, Amador R, Pérez-Torres D, Colón E, SánchezRivera C, Nieves-Plaza M, et al. Candidemia distribution, associated risk factors, and attributed mortality at a universitybased medical center. P R Health Sci J. 2010;29:26-9.

11. Marra AR, Camargo LF, Pignatari AC, Sukiennik T, Behar PR, Medeiros EA, et al. Nosocomial bloodstream infections in Brazilian hospitals: analysis of 2,563 cases from a prospective nationwide surveillance study. J Clin Microbiol. 2011;49:186671 .

12. Silva S, Negri M, Henriques M, Oliveira R, Williams DW, Azeredo J. Candida glabrata, Candida parapsilosis and Candida tropicalis: biology, epidemiology, pathogenicity and antifungal resistance. FEMS Microbiol Rev. 2012;36:288-305.

13. Pammi M, Holland L, Butler G, Gacser A, Bliss JM. Candida parapsilosis is a significant neonatal pathogen: a systematic review and meta-analysis. Pediatr Infect Dis J. 2013;32:E20616.

14. Branchini ML, Pfaller MA, Rhinechalberg J, Frempong T, Isenberg HD. Genotypic variation and slime production among blood and catheter isolates of Candida-parapsilosis. J Clin Microbiol. 1994;32:452-6.

15. Kuhn DM, Chandra J, Mukherjee PK, Ghannoum MA. Comparison of biofilms formed by Candida albicans and Candida parapsilosis on bioprosthetic surfaces. Infect Immun. 2002;70:878-88.

16. Trofa D, Gácser A, Nosanchuk JD. Candida parapsilosis, an emerging fungal pathogen. Clin Microbiol Rev. 2008;21:60625 .

17. Garner JS, Jarvis WR, Emori TG, Horan TC, Hughes JM. CDC definitions for nosocomial infections, 1988. Am J Infect Control. 1988;16:128-40.

18. Wisplinghoff H, Bischoff T, Tallent M, Seifert H, Wenzel RP, Edmond MB. Nosocomial bloodstream infections in US hospitals: analysis of 24,179 cases from a prospective nationwide surveillance study. Clin Infect Dis. 2004;39:309-17.

19. Marr KA, Seidel K, White TC, Bowden RA. Candidemia in allogeneic blood and marrow transplant recipients: evolution of risk factors after the adoption of prophylactic fluconazole. J Infect Dis. 2000;181:309-16.

20. Villalobos JM, Castro JA, Avilés A, Peláez MC, Somogyi T, Sandoval L. Candida parapsilosis: principal causa de candidemia en un hospital de referencia para adultos de Costa Rica. Rev Chilena Infectol. 2016;33:159-65.

21. Tosun I, Akyuz Z, Guler NC, Gulmez D, Bayramoglu G, Kaklikkaya N, et al. Distribution, virulence attributes and antifungal susceptibility patterns of Candida parapsilosis complex strains isolated from clinical samples. Med Mycol. 2013;51:483-92.

22. Almirante B, Rodriguez D, Cuenca-Estrella M, Almela M, Sanchez F, Ayats J, et al. Epidemiology, risk factors, and prognosis of Candida parapsilosis bloodstream infections: 
case-control population-based surveillance study of patients in Barcelona, Spain, from 2002 to 2003. J Clin Microbiol. 2006;44:1681-5.

23. Knaus WA, Draper EA, Wagner DP, Zimmerman JE. APACHE II: a severity of disease classification system. Crit Care Med. 1985;13:818-29.

24. Cuéllar-Cruz M, López-Romero E, Villagómez-Castro JC, RuizBaca E. Candida species: new insights into biofilm formation. Future Microbiol. 2012;7:755-71.

25. Ramage G, Rajendran R, Sherry L, Williams C. Fungal biofilm resistance. Int J Microbiol. 2012;2012:528521.

26. Fais R, Di Luca M, Rizzato C, Morici P, Bottai D, Tavanti A, et al. The N-Terminus of human lactoferrin displays anti-biofilm activity on Candida parapsilosis in lumen catheters. Front Microbiol. 2017;8:2218.
27. Douglas LJ. Candida biofilms and their role in infection. Trends Microbiol. 2003;11:30-6.

28. Shin JH, Kee SJ, Shin MG, Kim SH, Shin DH, Lee SK, et al. Biofilm production by isolates of Candida species recovered from nonneutropenic patients: comparison of bloodstream isolates with isolates from other sources. J Clin Microbiol. 2002;40:1244-8.

29. Panagoda GJ, Ellepola AN, Samaranayake LP. Adhesion of Candida parapsilosis to epithelial and acrylic surfaces correlates with cell surface hydrophobicity. Mycoses. 2001;44:29-35.

30. Kumar A. Systematic bias in meta-analyses of time to antimicrobial in sepsis studies. Crit Care Med. 2016;44:e234-5.

31. Levy MM, Evans LE, Rhodes A. The surviving sepsis campaign bundle: 2018 update. Crit Care Med. 2018;46:997-1000. 